\title{
Density reorganization in hot nuclei
}

\author{
S. K. Samaddar, ${ }^{1}$ J. N. De, ${ }^{1}$ X. Viñas, ${ }^{2}$ and M. Centelles ${ }^{2}$ \\ ${ }^{1}$ Saha Institute of Nuclear Physics, I/AF Bidhannagar, Kolkata 700064, India \\ ${ }^{2}$ Departament d'Estructura i Constituents de la Matèria, Facultat de Física, Universitat de Barcelona, \\ Diagonal 647, E-08028 Barcelona, Spain
}

(Received 24 January 2007; published 14 May 2007)

\begin{abstract}
The density profile of a hot nuclear system produced in intermediate-energy heavy ion collisions is studied in a microcanonical formulation with a momentum and density dependent finite range interaction. The caloric curve and density evolution with excitation are calculated for a number of systems for the equilibrium mononuclear configuration; they compare favorably with the recent experimental data. The studied density fluctuations are seen to build up rapidly beyond an excitation energy of $\sim 8 \mathrm{MeV} / \mathrm{u}$ indicating the instability of the system toward nuclear disassembly. Explicit introduction of deformation in the expansion path of the heated nucleus, however, shows that the system might fragment even earlier. We also explore the effects of the nuclear equation of state and of the mass and isospin asymmetry on the nuclear equilibrium configuration and the relevant experimental observables.
\end{abstract}

DOI: 10.1103/PhysRevC.75.054608

PACS number(s): 25.70.Pq, 25.70.Gh

\section{INTRODUCTION}

In intermediate-energy nuclear collisions, a hot nuclear system is initially produced in unstable equilibrium, generally in a compressed state. The unbalanced thermal and compressional pressure push the system toward expansion until an equilibrium configuration is reached. If the system is sufficiently excited (above a few $\mathrm{MeV}$ per nucleon), the mononuclear configuration is ultimately driven toward a subnuclear density (breakup density $\rho_{f}$ ) below which it no longer remains in the said configuration and undergoes disassembly into a number of fragments.

Study of the properties of hot nuclear systems at subnormal densities is of utmost physical interest in understanding explosive nucleosynthesis in supernova explosions [1,2], it plays a decisive factor in the element composition of heavenly bodies. Nuclear multifragmentation studies in the laboratory serve as a window for having a closer look at these processes; the properties of the diluted nuclear material prior to fragmentation have a key role in such an understanding. It further helps in mapping the nuclear equation of state (EOS) and also extracting, from the complex experimental data, the density dependence of the nuclear symmetry energy [3,4], which are crucial inputs in detailing supernova explosions and discerning the properties of neutron star matter.

From experimental and theoretical studies, it is seen that the breakup density $\rho_{f}$ decreases with increasing excitation energy. Since this density has a profile, use of a single value of it leaves some room for ambiguity. In experimental studies, it is understood as an average density. There are several experimental estimates of the breakup density. This has been determined from the studies of correlation functions of emitted light particles from the source [5], but the results seem to depend on the choice of the pair of correlated light fragments. It raises the question of the representation of the evolutionary stage of the reaction process as given by the correlation data, and thus there remains a latitude for considerable uncertainties. The systematics of the Coulomb barriers required to fit the intermediate mass ejectile spectra also give an estimate of the breakup densities [6]. However, in this extraction process, it is implicitly assumed that the barrier corresponds to a binary system [7], contrary to the fact that the hot system disassembles into many fragments. This introduces doubts in the proper estimation of the breakup densities. Recently, the breakup densities have also been derived from the analysis of apparent level density parameters required to fit the measured caloric curves [8]; the extraction, however, hinges on a suitable mix of theory [9] with experiment. The deduced breakup densities from different sets of experimental data sometimes have wide variations among them; a sound knowledge of these densities is thus still incomplete.

The disassembly of a mononuclear configuration at excitations above $\sim 3-4 \mathrm{MeV} / \mathrm{u}$ has been theoretically studied in a statistical framework for more than two decades. There has been a variety of these models [10,11], the basic underlying premise of which is that the excited nuclear system expands to a freeze-out volume and then the system undergoes a one-step prompt multifragmentation, the fragmentation pattern being determined by the available phase space. It is assumed that both thermal and chemical equilibria are established among the fragments at the freeze-out density $\rho_{f}$, so that the fragmentation pattern is frozen out there. In most of these models, the freeze-out density is independent of the excitation energy. Different models, however, use widely varying freezeout densities. Whereas in the canonical or microcanonical models $[10,11]$, the density $\rho_{f}$ is $\sim(0.12-0.2) \rho_{0}$ (where $\rho_{0}$ is the nuclear saturation density), the corresponding quantity in the lattice-gas model $[12,13]$ is $\sim(0.3-0.4) \rho_{0}$. This wide uncertainty in both the theoretical and experimental arena has recently prompted researchers to take a closer look at the dependence of nuclear density on excitation $[14,15]$. These calculations depend on the experimental premise that the hot systems formed in nuclear collisions are isolated systems having a fixed excitation energy (microcanonical ensemble); for equilibrium, they are driven to an expanded configuration with the maximum entropy. 
The calculations of Sobotka et al. [14] on nuclear expansion with excitation are schematic in nature; they employ a parametric form of the base density and then allow a selfsimilar expansion of the density to look for the maximum entropy configuration, the entropy being calculated in the Fermi-gas model. In the calculations reported in Ref. [15], the same physical picture was followed; however, a realistic effective Hamiltonian was used to calculate the base density profile in a Thomas-Fermi framework. This renders the base density self-consistent and allows one to calculate the entropy microscopically; furthermore, it offers the possibility of exploring the effect of the nuclear EOS by using a variety of effective interactions which, however, was not reported.

In Ref. [15], calculations were performed with the Skyrme interaction $\left(\mathrm{SkM}^{*}\right)$ for only one system, namely, ${ }^{150} \mathrm{Sm}$. For an expanding system pursuing the maximum entropy, the surface diffuseness is likely to play a key role [16], so a zero-range interaction like the Skyrme force may possibly not be suitable for studying such a density profile. It has been reported [17] that a constrained expanded system in a Thomas-Fermi approach leads to numerical instabilities, and the gradient (surface) terms in the energy density functional were replaced with a suitable Yukawa interaction [18]. In the present communication, we report calculations using the modified Seyler-Blanchard (SBM) interaction [19,20]; it is of finite range and is momentum and density dependent. Different sets of SBM parameters yield different nuclear EOSs. In the present communication, we have explored the role of nuclear EOS on the observables related to an expanded mononuclear equilibrium configuration. We have further studied the isospin and mass dependence of the observables. In addition to expansion, a possible deformation along the expansion path might contribute additional entropy and mimics a fragmentation channel. We have considered volume-conserving quadrupole deformation to explore this aspect.

\section{MODEL OVERVIEW}

The key tenets of the model have been reported in brief in Ref. [15]. In the following, we present a somewhat more comprehensive account of the theoretical framework used in the present calculation.

\section{A. Basic methodology}

In experimental conditions, when two nuclei collide at intermediate energy, a hot nuclear system with a fixed number of neutrons and protons is formed in a nonequilibrium state. The system might also be compressed initially, resulting in a collective flow in the decompression stage. We ignore the collective flow in the present calculation. As the system remains in isolation, the total excitation energy does not change in the subsequent evolution of the system, i.e., the system is microcanonical. To attain equilibrium, this hot system evolves in quest of maximum entropy. It is, however, still possible to describe the system statistically by an effective temperature $T$. Keeping the vestiges of a canonical temperature $T$ in the calculation has the operational advantage that it helps in defining an occupation function that can be employed in evaluating various observables like energy, entropy, etc. To obtain the equilibrium state, we adopted the following calculational procedure for a given excitation energy $E^{*}$ :

(i) The system is prepared in a finite temperature ThomasFermi (FTTF) method such that the thermal excitation energy $E_{\text {ther }}(T)=E(T)-E(T=0)$ is less than $E^{*}$. Here $E(T)$ is the total energy of this system at temperature $T$.

(ii) Keeping the temperature fixed at $T$, the system is allowed to undergo expansion until the total excitation (thermalplus-expansion) of the system equals $E^{*}$. The entropy for this configuration is evaluated.

(iii) We repeat the above steps with different initial temperatures $T$, and from the plethora of different configurations so obtained, the one with the maximum entropy represents the equilibrium mononuclear configuration.

In essence, this procedure aims at maximizing the entropy with respect to the collective coordinate that describes the expansion of the hot system under the constraint of constant excitation energy. Along the process, there is a transfer from the initial thermal energy of the system to the expansion energy in search of maximal entropy.

\section{B. Generation of the base density profile}

The FTTF framework is employed to generate the base density profile at temperature $T$. The effective interaction used for the calculation is taken to be the SBM interaction $[19,20]$ given by

$$
\begin{aligned}
v_{\mathrm{eff}}\left(\mathbf{r}_{1}, \mathbf{r}_{2}, p, \rho\right)= & -C_{l, u}\left[1-\frac{p^{2}}{b^{2}}-d^{2}\left\{\rho\left(\mathbf{r}_{1}\right)+\rho\left(\mathbf{r}_{2}\right)\right\}^{n}\right] \\
& \times \frac{\exp (-r / a)}{(r / a)} .
\end{aligned}
$$

Here $r=\left|\mathbf{r}_{1}-\mathbf{r}_{2}\right|$ and $p=\left|\mathbf{p}_{\mathbf{1}}-\mathbf{p}_{\mathbf{2}}\right|$ are the relative separation of the interacting nucleons in configuration and momentum space, $\rho\left(\mathbf{r}_{1}\right)$ and $\rho\left(\mathbf{r}_{2}\right)$ are the densities at the sites of these two nucleons, and $C_{l}$ and $C_{u}$ are the strengths for like pair $(n-n, p-p)$ and unlike pair $(n-p)$ interactions, respectively. Following Refs. $[19,20]$, the six parameters $C_{l}, C_{u}, a, b, d$, and $n$ are determined by reproducing the volume energy per particle of symmetric nuclear matter, its saturation density, volume asymmetry energy, surface energy, energy dependence of the real part of the nucleon-nucleus optical potential, and energies of isoscalar giant monopole resonances (ISGMR). The density exponent $n$ governs the nuclear EOS. To explore the effect of EOS on the relevant observables, we chose two sets of SBM parameters (given in Table I) with $n=1 / 6$ and $4 / 3$ that yield values of the nuclear incompressibility $K_{\infty}=238$ and $380 \mathrm{MeV}$ in infinite matter, respectively. The above interaction with $n=1 / 6$ reproduces quite well the ground-state binding energies, root-mean-square charge radii, and the ISGMR energies for a host of even-even nuclei varying from ${ }^{16} \mathrm{O}$ to very heavy systems. It has also been seen that for symmetric nuclear matter, the results obtained with this interaction agree very well $[21,22]$ with those calculated 
TABLE I. Parameters of effective interactions used in this paper (in $\mathrm{MeV} \mathrm{fm}$ ) and their bulk incompressibility modulus (in $\mathrm{MeV}$ ).

\begin{tabular}{lcccccc}
\hline \hline$n$ & $C_{l}$ & $C_{u}$ & \multicolumn{1}{c}{$a$} & $b$ & $d$ & $K_{\infty}$ \\
\hline $1 / 6$ & 291.7 & 910.6 & 0.6199 & 928.2 & 0.879 & 238 \\
$4 / 3$ & 210.7 & 653.2 & 0.529 & 615.4 & 0.924 & 380 \\
\hline \hline
\end{tabular}

microscopically with a realistic interaction in a variational approach [23,24].

The occupation function $n_{\tau}(\mathbf{r}, \mathbf{p}, T)$ for the finite system is obtained [20] from the minimization of the thermodynamic potential

$$
\Omega=E-T S-\sum_{\tau} \mu_{\tau} N_{\tau}
$$

It is given by

$$
\begin{aligned}
n_{\tau}(\mathbf{r}, \mathbf{p}, T)= & {\left[1+\exp \left\{\left(\frac{p^{2}}{2 m}+V_{\tau}^{0}(r)+p^{2} V_{\tau}^{1}(r)\right.\right.\right.} \\
& \left.\left.\left.+V_{\tau}^{2}(r)+\delta_{\tau, Z} V_{c}(r)-\mu_{\tau}\right) / T\right\}\right]^{-1},
\end{aligned}
$$

where $E$ and $S$ are the total energy and entropy of the nucleus, $N_{\tau}$ refers to the number of neutrons or protons ( $\tau$ is the isospin index), $\mu_{\tau}$ is their chemical potentials, and $m$ is their mass, taken to be the same for neutrons and protons. The quantities $V_{\tau}^{0}$ and $V_{\tau}^{1}$ are the components of the single-particle potentials corresponding to the momentum-independent and momentum-dependent parts of the interaction; $V_{\tau}^{2}$ is the rearrangement potential arising out of the density-dependent part of the interaction, and $V_{c}$ is the Coulomb potential which contains both the direct and the exchange parts. Expressions for the different components of the single-particle potential are given in Appendix A. The momentum-dependent part $V_{\tau}^{1}$ determines the nucleon $k$ mass $m_{\tau, k}(r)$ as

$$
\frac{p^{2}}{2 m_{\tau, k}(r)}=\frac{p^{2}}{2 m}+p^{2} V_{\tau}^{1}(r)
$$

The effective single-particle (SP) potential $\mathcal{V}_{\tau}(r)$ is given by

$$
\mathcal{V}_{\tau}(r)=V_{\tau}^{0}(r)+V_{\tau}^{2}(r)+\delta_{\tau, Z} V_{c}(r) .
$$

The occupation function given by Eq. (3) is then rewritten as

$n_{\tau}(\mathbf{r}, \mathbf{p}, T)=\left[1+\exp \left\{\left(\frac{p^{2}}{2 m_{\tau, k}(r)}+\mathcal{V}_{\tau}(r)-\mu_{\tau}\right) / T\right\}\right]^{-1}$.

The base density is obtained from

$$
\rho_{\tau}(r)=\frac{2}{h^{3}} \int n_{\tau}(\mathbf{r}, \mathbf{p}, T) d \mathbf{p}=A_{T}^{*}(r) J_{1 / 2}\left(\eta_{\tau}(r)\right),
$$

where

$$
A_{T}^{*}(r)=\frac{4 \pi}{h^{3}}\left[2 m_{\tau, k}(r) T\right]^{3 / 2},
$$

and $J_{K}\left(\eta_{\tau}\right)$ is the Fermi integral

$$
J_{K}\left(\eta_{\tau}\right)=\int_{0}^{\infty} \frac{x^{K}}{1+\exp \left(x-\eta_{\tau}\right)} d x,
$$

with the fugacity $\eta_{\tau}$ given as

$$
\eta_{\tau}(r)=\left[\mu_{\tau}-\mathcal{V}_{\tau}(r)\right] / T .
$$

The occupation probability $n_{\tau}(\mathbf{r}, \mathbf{p}, T)$ given by Eq. (6) can also be expressed as the SP occupancy in the energy space as

$$
f\left(\varepsilon_{\tau}, \mu_{\tau}, T\right)=\left[1+\exp \left\{\left(\varepsilon_{\tau}-\mu_{\tau}\right) / T\right\}\right]^{-1},
$$

with the SP energy written as

$$
\varepsilon_{\tau}=\frac{p^{2}}{2 m_{\tau, k}(r)}+\mathcal{V}_{\tau}(r) .
$$

The base density from Eq. (7) can be recast, in terms of the SP potential and energy, as

$$
\begin{aligned}
\rho_{\tau}(r)= & \frac{1}{2 \pi^{2}}\left[\frac{2 m_{\tau, k}(r)}{\hbar^{2}}\right]^{\frac{3}{2}} \\
& \times \int_{\mathcal{V}_{\tau}(r)}^{\infty} \sqrt{\varepsilon_{\tau}-\mathcal{V}_{\tau}(r)} f\left(\varepsilon_{\tau}, \mu_{\tau}, T\right) d \varepsilon_{\tau} .
\end{aligned}
$$

This density profile is generated self-consistently in an iterative procedure. The chemical potentials $\mu_{\tau}$ are determined from the nucleon number conservation:

$$
\begin{aligned}
N_{\tau} & =\int \rho_{\tau}(r) d \mathbf{r} \\
& =\int g_{\tau}\left(\varepsilon_{\tau}, T\right) f\left(\varepsilon_{\tau}, \mu_{\tau}, T\right) d \varepsilon_{\tau},
\end{aligned}
$$

where the single-particle level density $g_{\tau}\left(\varepsilon_{\tau}, T\right)$ is given by

$$
g_{\tau}\left(\varepsilon_{\tau}, T\right)=\frac{4 \sqrt{2}}{\pi \hbar^{3}} \int\left(m_{\tau, k}(r)\right)^{\frac{3}{2}} \sqrt{\varepsilon_{\tau}-\mathcal{V}_{\tau}(r)} r^{2} d r .
$$

The modeling of a hot finite nuclear system poses some problems as it is thermodynamically unstable. The main difficulty arises in taking proper account of the continuum states which are occupied at a finite temperature as a result of which the particle density does not vanish at large distances. The extracted observables then depend on the size of the box in which the calculations are performed. This problem is overcome in the so-called subtraction procedure $[25,26]$ where the hot nucleus, assumed to be a thermalized system in equilibrium with a surrounding gas representing evaporated nucleons, is separated from its embedding environment. The method is based on the existence of two solutions to the finite temperature Thomas-Fermi equations, one corresponding to the liquid phase with the surrounding gas $(l g)$ and the other corresponding to the gas $(g)$ phase. These two solutions are obtained from the variational equations

$$
\frac{\delta \Omega_{l g}}{\delta \rho_{\tau, l g}}=0,
$$

and

$$
\frac{\delta \Omega_{g}}{\delta \rho_{\tau, g}}=0,
$$

where $\Omega_{l g}$ and $\Omega_{g}$ are the thermodynamic potentials [as defined in Eq. (2)] of the respective systems with the same chemical potentials. The base density profile for the hot nucleus in question is given by $\rho_{\tau}=\rho_{\tau, l g}-\rho_{\tau, g}$ (which may also be called the liquid profile); this is independent of the box 
size in which calculations are done. The density $\rho_{\tau}$ goes to zero at large distances, implying a vanishing surface pressure.

The expressions for $\rho_{l g}(r)$ and $\rho_{g}(r)$ when written in terms of the SP potential and energy are analogous to that given in Eq. (13). Obviously, the nucleon $k$ mass and the SP potentials in the two phases are different, and

$$
\int\left[\rho_{\tau, l g}(r)-\rho_{\tau, g}(r)\right] d \mathbf{r}=N_{\tau} .
$$

\section{Expansion of the system}

The density profile of an expanded system, in principle, can be treated in a constrained Thomas-Fermi procedure [17]. However, for large expansions, instability sets in which is amplified in the subtraction procedure for the extraction of the bloated nuclear density. We, therefore, simulate the expansion of the system through a self-similar scaling approximation for the density, that is,

$$
\rho_{\lambda}(r)=\lambda^{3} \rho(\lambda r)
$$

where the scaling parameter $\lambda$ is unity for the unbloated nucleus and decreases with expansion, lying in the range $0<\lambda \leqslant 1 ; \rho_{\lambda}(r)$ is the scaled density and $\rho(r)$ is the base density profile generated in the FTTF framework.

Besides its simplicity, there is no a priori reason for choosing the self-similar expansion. However, some justification can be found on the grounds that in a simplistic situation with a harmonic oscillator potential at small temperatures, the scaled and constrained density profiles are equivalent. This is shown in Appendix B.

\section{Effect of correlations on density}

The self-consistent Thomas-Fermi procedure generates the single-particle (mean field) potential; it does not include the coupling of the single-particle motion with the collective degrees of freedom [27]. This coupling introduces an extra energy dependence in the effective mass of the nucleons (in addition to the contribution from the momentum dependence in the effective interaction). Incorporating this effect, the total effective mass $m^{*}$ (the effective mass is position dependent; from now on, this dependence is not explicitly shown) is then defined as

$$
m^{*}=m \frac{m_{k}}{m} \frac{m_{\omega}}{m}
$$

where $m_{\omega}$ (the $\omega$ mass) is the energy or frequency dependent part of the effective mass. The $\omega$ mass is surface peaked and has values generally larger [28] than the nucleon mass. This increased effective mass has the effect of bringing down the excited states from higher energy to lower energy near the Fermi surface, thus increasing the many-body density of states at low excitations. The system can then accommodate comparatively more entropy at a given excitation energy; in the present case, in searching for the state with maximum entropy, these correlations might have a significant role to play. An $a b$ initio determination of the $\omega$ mass [28,29] as a function of temperature is very involved; we have therefore taken a phenomenological form [30-32] for $m_{\omega}$ such that

$$
\begin{aligned}
\frac{m_{\omega}}{m}= & 1-0.4(\mathrm{fm}) A^{\frac{1}{3}} \exp \left[-\left(\frac{T}{21(\mathrm{MeV}) A^{-\frac{1}{3}}}\right)^{2}\right] \\
& \times \frac{1}{\rho(0)} \frac{d \rho(r)}{d r}
\end{aligned}
$$

where $\rho(0)$ is the central density of the density distribution, temperature $T$ is measured in $\mathrm{MeV}$, and $m$ is the nucleon mass. The collectivity as introduced refers to the liquid phase only; the density in the above equation is then $\rho(r)=\rho^{\lg }(r)-$ $\rho^{g}(r)$, and $A$ refers to the liquid mass. We have thus taken the $\omega$ mass of the liquid-plus-gas phase as $m_{\omega}^{l g}=m_{\omega}$, the $\omega$ mass of the liquid; for the gas phase, the frequency dependence in the effective mass is neglected, i.e., $m_{\omega}^{g}=m$.

A self-consistent calculation of the density profile with the inclusion of $\omega$ mass is extremely complex; to avoid the complexities, we have therefore adopted the following approach, which is a realistic extension of the method given in Ref. [31]. For each fixed temperature $T$, we assume that the nucleus is well described by the mean field and the effective mass given by Eq. (20). The self-energy of a particle in the nuclear medium is usually approximated by a local field $\left(m^{*} / m\right) U(r)$, where $U(r)$ is a local potential (for instance, a Woods-Saxon potential). In our case, $m_{k}$ is explicitly included in the self-consistently generated $\mathcal{V}(r)$. Therefore, due to the frequency dependence in the effective mass, $g(\varepsilon)$ as defined in Eq. (15) is modified, taking into account the subtraction procedure, as

$$
\begin{aligned}
\tilde{g}_{\tau}\left(\varepsilon_{\tau}, T\right)= & \frac{4 \sqrt{2}}{\pi \hbar^{3}} \int\left[\left(m_{\tau, k}^{l g} \frac{m_{\omega}}{m}\right)^{\frac{3}{2}} \sqrt{\varepsilon_{\tau}-\mathcal{V}_{\tau}^{l g}(r) \frac{m}{m_{\omega}}}\right. \\
& \left.-\left(m_{\tau, k}^{g}\right)^{\frac{3}{2}} \sqrt{\varepsilon_{\tau}-\mathcal{V}_{\tau}^{g}(r)}\right] r^{2} d r .
\end{aligned}
$$

In Eq. (22), the first term in the square bracket corresponds to the liquid-plus-gas part and the second term corresponds to the gas part.

The chemical potential $\mu_{\tau}$ is now modified to $\tilde{\mu}_{\tau}$ to conserve the particle number:

$$
N_{\tau}=\int \tilde{g}_{\tau}\left(\varepsilon_{\tau}, T\right) f\left(\varepsilon_{\tau}, \tilde{\mu}_{\tau}, T\right) d \varepsilon_{\tau} .
$$

The expressions for the densities are also accordingly modified:

$$
\begin{aligned}
\tilde{\rho}_{\tau}^{i}(r)= & \frac{1}{2 \pi^{2} \hbar^{3}}\left[2 m_{\tau, k}^{i} \frac{m_{\omega}^{i}}{m}\right]^{3 / 2} \\
& \times \int \sqrt{\varepsilon_{\tau}-\mathcal{V}_{\tau}^{i} \frac{m}{m_{\omega}^{i}}} f\left(\varepsilon_{\tau}, \tilde{\mu}_{\tau}, T\right) d \varepsilon_{\tau},
\end{aligned}
$$

where $i$ refers to $l g$ or $g$.

In the present context, the following prescription is adopted to include the effect of correlations on the scaled density $\rho_{\lambda}$; the single-particle potential $\mathcal{V}_{\lambda}$ and the $k$ mass $m_{k, \lambda}$ are evaluated for the scaled density, the $\omega$ mass $m_{\omega, \lambda}$ is calculated from this density according to Eq. (21), and then the single-particle level density $\tilde{g}_{\lambda}$ and the chemical potential $\tilde{\mu}_{\lambda}$ are generated by using Eqs. (22) and (23) as already stated. This gives the correlated scaled density $\tilde{\rho}_{\lambda, \tau}^{i}(r)$. 


\section{E. Energy and entropy}

In the absence of correlations, the total energy of the finite unexpanded nucleus is expressed in terms of the uncorrelated density and the occupancy given by Eqs. (13) and (6). The total energy density is obtained as

$$
\mathcal{E}(r)=\sum_{\tau}\left[\mathcal{E}_{\tau}^{\mathrm{kin}}(r)+\mathcal{E}_{\tau}^{\mathrm{int}}(r)\right]
$$

where $\mathcal{E}_{\tau}^{\text {kin }}(r)$ and $\mathcal{E}_{\tau}^{\text {int }}(r)$ are the kinetic energy and the interaction energy density, respectively. The kinetic energy density is

$$
\begin{aligned}
\mathcal{E}_{\tau}^{\mathrm{kin}}(r) & =\frac{2}{h^{3}} \int \frac{p^{2}}{2 m} n_{\tau}(\mathbf{r}, \mathbf{p}) d \mathbf{p} \\
& =\frac{2 \pi}{m h^{3}}\left(2 m_{\tau, k} T\right)^{5 / 2} J_{3 / 2}\left(\eta_{\tau}(r)\right),
\end{aligned}
$$

where the effective mass $m_{\tau, k}$ and the Fermi integral $J_{K}(\eta)$ are defined through Eqs. (4) and (9), respectively.

The interaction energy density is given by

$$
\begin{aligned}
\mathcal{E}_{\tau}^{\text {int }}(r)= & \frac{1}{2}\left[V_{\tau}^{0}(r) \rho_{\tau}(r)+\frac{2}{h^{3}} V_{\tau}^{1}(r) \int p^{2} n_{\tau}(\mathbf{r}, \mathbf{p}) d \mathbf{p}\right] \\
& +\mathcal{E}_{c}(r) \delta_{\tau, Z} \\
= & \frac{1}{2} V_{\tau}^{0}(r) \rho_{\tau}(r)+\frac{2 \pi}{h^{3}}\left(2 m_{\tau, k} T\right)^{5 / 2} J_{3 / 2}\left(\eta_{\tau}(r)\right) V_{\tau}^{1}(r) \\
& +\mathcal{E}_{c}(r) \delta_{\tau, Z} .
\end{aligned}
$$

The Coulomb energy density $\mathcal{E}_{c}(r)$ is the sum of the direct and exchange contributions:

$$
\mathcal{E}_{c}(r)=\mathcal{E}_{c}^{D}(r)+\mathcal{E}_{c}^{\mathrm{Ex}}(r) .
$$

The direct term is obtained as

$$
\mathcal{E}_{c}^{D}(r)=\pi e^{2} \frac{\rho_{p}(r)}{r} \int_{0}^{\infty} \rho_{p}\left(r^{\prime}\right)\left[\left(r+r^{\prime}\right)-\left|r-r^{\prime}\right|\right] r^{\prime} d r^{\prime},
$$

and the exchange term is calculated from the Slater approximation as

$$
\mathcal{E}_{c}^{\mathrm{Ex}}(r)=-\frac{3 e^{2}}{4 \pi}\left(3 \pi^{2}\right)^{1 / 3} \rho_{p}^{4 / 3}(r) .
$$

Combining Eqs. (26) and (27), one may write Eq. (25) as

$$
\begin{aligned}
\mathcal{E}(r)= & \sum_{\tau}\left[\frac{2 \pi}{h^{3}}\left(2 m_{\tau, k} T\right)^{5 / 2} J_{3 / 2}\left(\eta_{\tau}(r)\right)\left(\frac{1}{m}+V_{\tau}^{1}\right)\right. \\
& \left.+\frac{1}{2} V_{\tau}^{0}(r) \rho_{\tau}(r)+\mathcal{E}_{c}(r) \delta_{\tau, Z}\right] .
\end{aligned}
$$

The total energy is

$$
E=\int \mathcal{E}(r) d \mathbf{r} .
$$

The total entropy in the Landau quasiparticle approximation for the nuclear system with the base density profile is

$$
S=-\sum_{\tau} \int g_{\tau}\left(\varepsilon_{\tau}, T\right)[f \ln f+(1-f) \ln (1-f)] d \varepsilon_{\tau},
$$

where the SP level density and occupancy are given by Eqs. (15) and (11).
The energy of the expanded correlated system is calculated with the suitably modified effective mass, single-particle potentials and fugacity. The entropy of the correlated system can be similarly calculated. In the subtraction procedure, the energy $E$ of the nuclear liquid is obtained through

$$
E=E_{l g}-E_{g},
$$

where $E_{l g}$ and $E_{g}$ are the total energies of the liquid-plus-gas system and of the gas alone; these energies are calculated from Eq. (31) with appropriate modification for expansion and correlation effects. For the entropy, the subtracted SP level density of the form given by Eq. (22) (which includes correlation effects) but modified for scaling is employed.

\section{F. Deformation of the system}

So far, we have taken the shape of the hot expanding system to be spherical. In search of the maximum entropy configuration, however, the system may explore possible deformation paths along with expansion mimicking fragmentation. For simplicity, we consider only a volume-conserving quadrupole (spheroidal) deformation at all stages of expansion and check whether there is additional gain in entropy from deformation of the system.

In a volume-conserving deformation, only the surface and Coulomb energies change. To calculate these changes, a sharp surface approximation to the density profile is made with $R_{\text {sharp }}=\sqrt{\frac{5}{3}\left\langle r^{2}\right\rangle}$. This also facilitates the calculation of the entropy gain from deformation. The excess Coulomb energy $\delta E_{c}(\lambda, \beta)$ of the expanded deformed system is given by [33]

$$
\delta E_{c}(\lambda, \beta)=E_{c}(\lambda, 0)\left[(1-x)^{1 / 3}|x|^{-1 / 2} F(x)-1\right],
$$

where $\beta$, the deformation of the spheroidal system in terms of the lengths $2 a$ and $2 c$ of its principal axes, is defined as

$$
\beta=\frac{4}{3} \ln (c / a),
$$

where $a<c$ for prolate and $a>c$ for oblate spheroids. The constraint of volume conservation gives $a^{2} c=R_{\text {sharp }}^{3}$. The quantity $x$, in terms of $\beta$, takes the form

$$
x=\frac{3}{2} \beta \frac{(1+\beta / 8)}{(1+\beta / 2)^{2}},
$$

and the function $F(x)$ reads

$$
\begin{aligned}
F(x) & =\frac{1}{2}[\ln (1+\sqrt{x})-\ln (1-\sqrt{x})], \quad \text { for } a<c, \\
& =\tan ^{-1}|x|^{1 / 2}, \quad \text { for } a>c .
\end{aligned}
$$
is

The expression of the surface area of the deformed nucleus

$$
\begin{aligned}
\mathcal{A}(\lambda, \beta)= & 2 \pi a^{2}+\frac{2 \pi a c^{2}}{\sqrt{c^{2}-a^{2}}} \sin ^{-1}\left(\frac{\sqrt{c^{2}-a^{2}}}{c}\right), \text { for } a<c \\
= & \frac{\pi}{\sqrt{a^{2}-c^{2}}}\left[2 a^{2} \sqrt{a^{2}-c^{2}}+a c^{2}\right. \\
& \left.\times \ln \left(\frac{a+\sqrt{a^{2}-c^{2}}}{a-\sqrt{a^{2}-c^{2}}}\right)\right], \quad \text { for } a>c .
\end{aligned}
$$


The change in surface free energy due to deformation is given by

$$
\delta F(\lambda, \beta)=\delta \mathcal{A}(\lambda, \beta) \sigma(\rho, X, T),
$$

where $\delta \mathcal{A}$ is the excess surface area due to deformation. The surface tension coefficient $\sigma$ is density, temperature, and asymmetry dependent; it is taken as

$$
\sigma(\rho, X, T)=\sigma\left(\rho_{0}, X, T=0\right) y(\rho) \alpha(T),
$$

with $\sigma\left(\rho_{0}, X, T=0\right)=\sigma\left(\rho_{0}, X=0, T=0\right)-a_{s} X^{2}$; here $a_{s}$ is the surface asymmetry coefficient taken to be $1.7826 \mathrm{MeV} \mathrm{fm} \mathrm{fm}^{-2}, X=(N-Z) / A$ is the asymmetry parameter of the nucleus, and $\sigma\left(\rho_{0}, X=0, T=0\right)=$ $1.06 \mathrm{MeV} \mathrm{fm}^{-2}$ is the surface energy coefficient of symmetric semi-infinite nuclear matter.

The surface tension has its maximum value at the saturation density $\rho_{0}$. The density dependence of the surface tension coefficient is evaluated by applying the scaling approximation on the ground-state density profile of semi-infinite nuclear matter. In the subnuclear density region, it is found to be well approximated by a polynomial $y(\rho)$ of the form

$$
y(\rho)=\sum_{k=1}^{4} b_{k}\left(\rho / \rho_{0}\right)^{k} .
$$

The values of the $b_{k}$ coefficients are $b_{1}=0.5382, b_{2}=$ $2.3124, b_{3}=-2.2312$, and $b_{4}=0.3816$. The temperature dependence of the surface tension is taken as [35]

$$
\alpha(T)=\left(1+\frac{3}{2} \frac{T}{T_{c}}\right)\left(1-\frac{T}{T_{c}}\right)^{3 / 2},
$$

with $T_{c}=15 \mathrm{MeV}$, the critical temperature for nuclear matter.

The excess entropy from deformation is calculated as $\delta S=-\partial(\delta F) /\left.\partial T\right|_{\rho}$. The excess surface energy is obtained from $\delta E_{\text {surf }}=\delta F+T \delta S$. The total energy of the expanded deformed system can then be written as

$$
E(\lambda, \beta, T)=E(\lambda, 0, T)+\delta E_{c}(\lambda, \beta, T)+\delta E_{\text {surf }}(\lambda, \beta, T) .
$$

The excitation energy is given by

$$
E^{*}(\lambda, \beta, T)=E(\lambda, \beta, T)-E(\lambda=1, \beta=0, T=0),
$$

and the corresponding total entropy is

$$
S(\lambda, \beta, T)=S(\lambda, 0, T)+\delta S(\lambda, \beta, T) .
$$

\section{RESULTS AND DISCUSSIONS}

To explore the density reorganization in nuclei with increasing excitation energy, we chose three isobars of mass $A=150$ (namely, $\mathrm{Cs}, \mathrm{Sm}$, and $\mathrm{Yb}$ ) and two isobars of mass $A=40(\mathrm{Ca}$ and $\mathrm{S})$ as representative systems. The two different sets of isobars with different isospins are chosen in order to study the mass as well as the asymmetry dependence on the evolution of density and some other characteristic observables related to the mononuclear configuration. Two sets of SBM interaction parameters (given in Table I) with widely varying nuclear incompressibility are used to investigate the effect

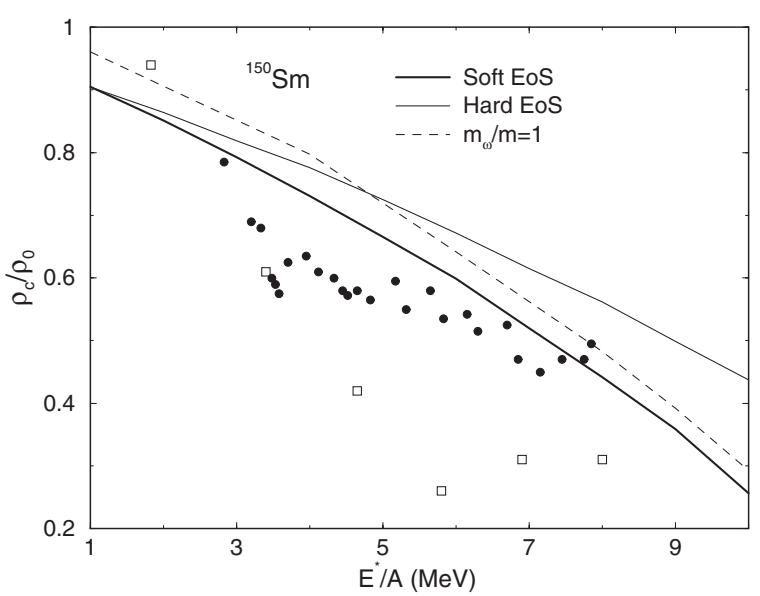

FIG. 1. Calculated equilibrium density of ${ }^{150} \mathrm{Sm}$ as a function of excitation energy per nucleon. Thick line corresponds to the calculations with the soft EOS $\left(K_{\infty}=238 \mathrm{MeV}\right)$; thin line, to those with the hard EOS ( $\left.K_{\infty}=380 \mathrm{MeV}\right)$; dashed line, to calculations without correlation effects for the softer EOS. Filled circles are the experimental data from Ref. [8]; open squares, from Ref. [6].

of the nuclear EOS on the observables considered. All the calculations reported are performed at different fixed excitation energies with the inclusion of thermal and expansion effects and the incorporation of the correlation effects introduced through the frequency dependence of the nucleon effective mass.

The dependence of the observables related to the excited mononuclear configuration on the nuclear EOS has not been studied so far. In Fig. 1, the density evolution of the hot $\mathrm{Sm}$ nucleus is displayed as a function of the excitation energy $E^{*} / A$ for two different EOSs, one softer with $K_{\infty}=$ $238 \mathrm{MeV}$ and the other one harder with $K_{\infty}=380 \mathrm{MeV}$. Here, the quantity $\rho_{c}$ represents the central density of the hot expanded nucleus in equilibrium corresponding to the maximum entropy configuration, and $\rho_{0}$ is the ground-state central density evaluated in the self-consistent Thomas-Fermi framework. Obviously, at zero excitation, $\rho_{c} / \rho_{0}=1$. It is found that for the softer EOS, above $E^{*} / A \sim 11 \mathrm{MeV}$, the entropy increases monotonically with expansion, and hence the mononuclear configuration ceases to exist; for the harder EOS, this limiting excitation energy is somewhat higher. At a given excitation, the equilibrium density is seen to be lower for the softer EOS. This can be understood from the following qualitative argument: the maximum entropy configuration is an outcome of the balance between the entropy gain from expansion and a loss due to reduction in temperature. For a harder EOS, the gain in entropy from expansion is energetically costlier for a given excitation energy; therefore, the expansion is less for a harder EOS. A more quantitative explanation in a simplistic model is given in Appendix C. The effects of correlation on the mononuclear observables with increasing excitation energy for a Skyrme-type $\left(\mathrm{SkM}^{*}\right)$ force have been discussed in an earlier paper [15]. It was found that the changes in the relevant observables with the inclusion of correlation effects with the SBM force are qualitatively the same as the $\mathrm{SkM}^{*}$ interaction, and therefore we do not want to 
delve into these details. However, for completeness, we present some selective results only for the nucleus ${ }^{150} \mathrm{Sm}$. In Fig. 1, the dashed curve displays the results for the evolution of central density without correlation effects for the softer SBM force. As in Ref. [15], the effect of correlation is found to reduce the central density at all excitations considered.

Two sets of experimentally derived data are also shown in Fig. 1; the filled circles are the experimental points extracted from the apparent level density parameters [8] for the mass selection $140<A<180$ and the open squares are the ones obtained from the Coulomb barrier systematics [6] for Aulike systems. It is found that the calculated results with the softer EOS having nuclear incompressibility very close to the generally accepted value [36] compare favorably well with those derived from the level density parameters.

In the following, the calculations are done with the softer EOS, and the reported results are to be understood in its terms, unless otherwise mentioned. In the upper panel of Fig. 2, the density $\rho_{c}$ evolution with excitation for the three nuclei Cs, $\mathrm{Sm}$, and $\mathrm{Yb}$ is shown along with the experimental data $[6,8]$. Since the density has a profile, its characterization by a single entity $\rho_{c}$ leaves some room for ambiguity. We have therefore also considered an average density $\rho=A / V_{\text {eq }}$ where $V_{\text {eq }}$ is the effective volume of the excited nucleus in equilibrium, taken as $V_{\text {eq }}=\frac{4}{3} \pi R_{\text {sharp }}^{3}$, where the sharp surface radius $R_{\text {sharp }}$ is calculated as $R_{\text {sharp }}=\sqrt{\frac{5}{3}\left\langle r^{2}\right\rangle}$ from the density distribution. In the lower panel of the figure, the density ratios are displayed as $V_{0} / V_{\text {eq }}$ as a function of excitation energy per nucleon. Here $V_{0}$ is the ground-state effective volume. Inspection of the two panels shows that the two sets of density ratios so defined are somewhat different. The difference is more manifest at higher excitations. The experimental data obtained from the apparent level density parameters lie in between the two sets of calculated densities.

Figure 2 displays the asymmetry dependence of the density evolution with excitation. It is seen that the more symmetric nucleus has generally a higher equilibrium density at a

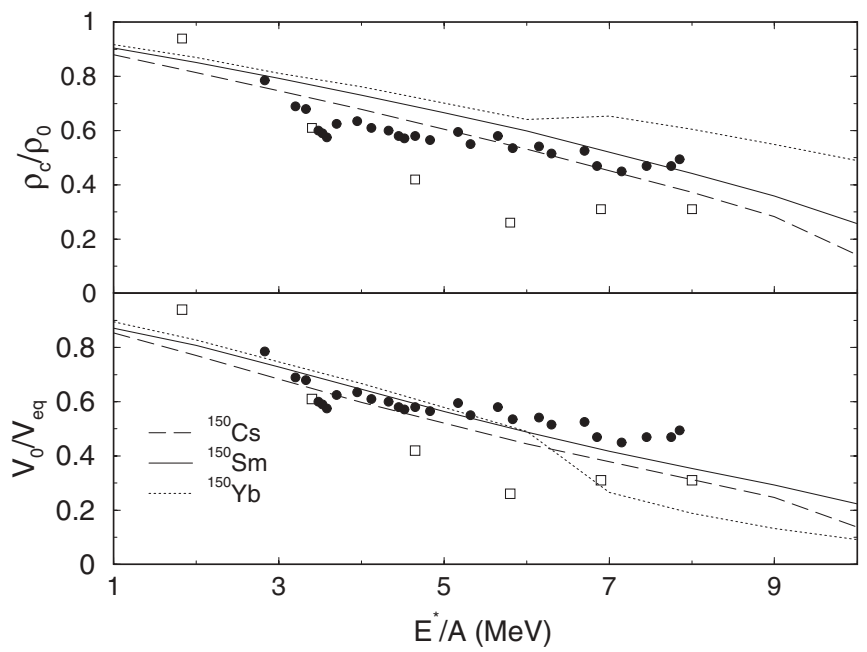

FIG. 2. Calculated equilibrium densities as a function of excitation energy for $A=150$ isobars in the two definitions of density as explained in the text. Experimental points are same as in Fig. 1. given excitation. This may be understood by examining the asymmetry dependence of the incompressibility $K_{A}$ of finite nuclei. It can be written in powers of $A^{-1 / 3}$ with a liquid-drop type expansion [36-38]:

$$
K_{A}=K_{v}+K_{s} A^{-1 / 3}+K_{\delta} \delta^{2}+K_{c} Z^{2} A^{-4 / 3}+\cdots .
$$

Here $K_{v}, K_{s}, K_{\delta}$, and $K_{c}$ are the volume, surface, asymmetry, and Coulomb coefficients, respectively, and $\delta$ is the asymmetry parameter $\delta=(N-Z) / A$. For isobars, the asymmetry and Coulomb terms are different. From the known [37,38] values of $K_{\delta}$ and $K_{c}$ (which are negative), it is found that the more symmetric nucleus has a larger incompressibility $K_{A}$, and thus, in the context of the discussions in relation to Fig. 1, it follows that the equilibrium density of the more symmetric nucleus is larger. For the nucleus with the largest charge considered here, there is a marked deviation from the smooth decrease of density with excitation in the energy range $6<E^{*} / A<8 \mathrm{MeV}$; it is difficult to discern the exact reason for it because of the delicate interplay of the Coulomb, thermal, and expansion effects on the density distribution. The manifestly opposite behavior of the density ratios as defined in the two panels of Fig. 2 can be understood by examining the density distributions at the relevant excitations as given in Fig 3. Particularly noticeable is the inversion of the trend of the central density with excitation at $E^{*} / A=7 \mathrm{MeV}$. The steep decrease of the density ratio given by $V_{0} / V_{\text {eq }}$ can be understood from the rapid growth of the density tail with excitation. The evolution of density with excitation energy for the $A=40$ systems ( $\mathrm{Ca}$ and $\mathrm{S}$ ) is displayed in Fig. 4. As in the case of heavier systems explored (Fig. 2), the density for the more symmetric system is higher; the asymmetry effect on the density is seen to be more prominent for the heavier systems. It is further found that for the same asymmetry, the heavier systems have somewhat higher equilibrium density at a given excitation. Note that the two definitions used for the characterization of density yield values that are not much different from the values for the lighter systems.

The expansion of the nucleus has an important bearing on the correlation of excitation energy with temperature. The

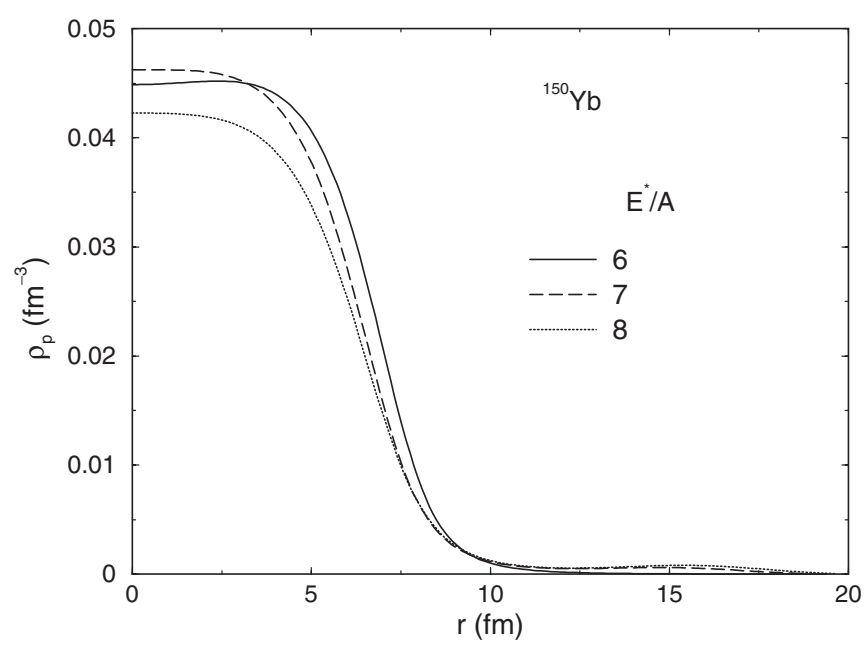

FIG. 3. Equilibrium proton density profile for the system ${ }^{150} \mathrm{Yb}$ at excitation energies of 6,7 , and $8 \mathrm{MeV}$ per nucleon. 


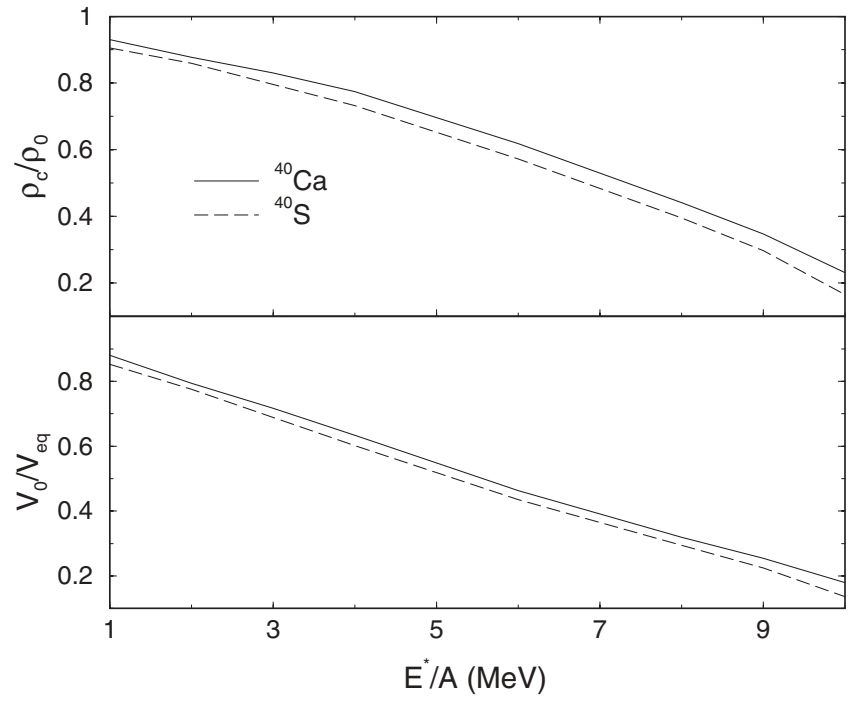

FIG. 4. Same as in Fig. 2, but for the nuclei ${ }^{40} \mathrm{Ca}$ and ${ }^{40} \mathrm{~S}$.

correlation (caloric curve) so obtained for the expanded hot nucleus ${ }^{150} \mathrm{Sm}$ is displayed in Fig. 5. At a fixed excitation energy, the system cools down with expansion; therefore the recorded temperature at the equilibrium configuration is significantly lower than that for the unexpanded nucleus prepared initially with the same excitation. In all of our calculations, the temperature refers to the canonical temperature. We have checked that the microcanonical temperature obtained from $T^{-1}=\partial S_{\text {eq }} / \partial E^{*}$ is not much different from the canonical one. To explore the role of the EOS on the correlation between $E^{*}$ and $T$, the caloric curves obtained from both the softer $\left(K_{\infty}=\right.$ $238 \mathrm{MeV})$ and the harder $\left(K_{\infty}=380 \mathrm{MeV}\right)$ EOS are shown in Fig. 5. It is seen that at a given $E^{*}$, the equilibrium temperature is significantly higher, particularly at high excitations, for the stiffer EOS. Tentatively, this can be understood from the fact

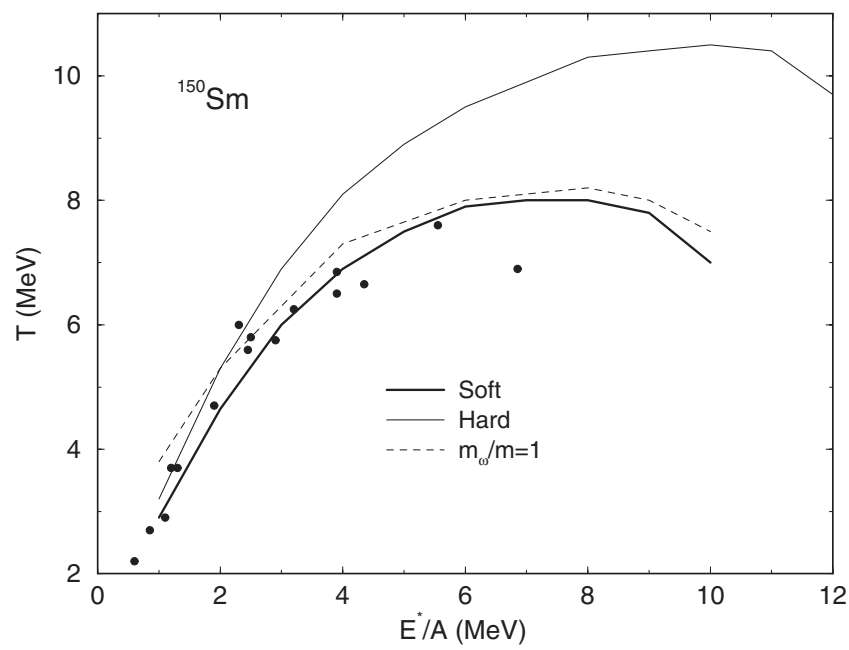

FIG. 5. Mononuclear caloric curve for the system ${ }^{150} \mathrm{Sm}$. Thick and thin lines refer to soft and hard EOS, respectively. Dashed line corresponds to the caloric curve calculated without correlation effects for the softer EOS. Experimental data points (filled circles) are from Ref. [39]. that the equilibrium density is higher for a harder EOS (Fig. 1), the corresponding expansion is less, and so more energy is locked in the thermal mode with a resultant higher temperature. The same conclusion is arrived at from the simplistic Fermi-gas type framework presented in Appendix C. A representative set of experimental data [39] for medium-mass nuclei are also shown in the figure. It is seen that the calculated caloric curve with the softer EOS compares very well with the experimental data. The dashed curve represents the caloric curve without inclusion of correlation effects $\left(m_{\omega} / m=1\right)$. As in Ref. [15], the introduction of correlation reduces the temperature for the equilibrium configuration at all excitation energies considered.

The dependence of the nuclear caloric curve on mass and isospin asymmetry is shown in Fig. 6. The top panel refers to the heavier isobars, and the bottom panel to the lighter ones. It is apparent that the caloric curves are nearly independent of asymmetry at lower excitations. For $E^{*} / A>5 \mathrm{MeV}$, the isospin dependence becomes increasingly prominent with increasing excitation, and the equilibrium temperature is higher for the more symmetric system. The mass dependence on the caloric curve is seen from an examination of the results for pairs of nuclei with almost the same asymmetry, such as $\mathrm{Sm}$ and $\mathrm{S}$ or $\mathrm{Yb}$ and $\mathrm{Ca}$. It is found that the equilibrium temperature for the lighter nuclei are comparatively always lower at all excitations. This behavior of the caloric curve with mass and isospin can be explained [using Eq. (47)] from the fact that for the same asymmetry, heavier nuclei have larger incompressibility; also, for isobars, the ones with more asymmetry have an effectively lower incompressibility.

The caloric curves show an interesting feature, namely, the occurrence of negative heat capacity, generally beyond an excitation energy $E^{*} / A \sim 8 \mathrm{MeV}$. Intuitively, one understands that if a system with a given excitation expands, it does so at

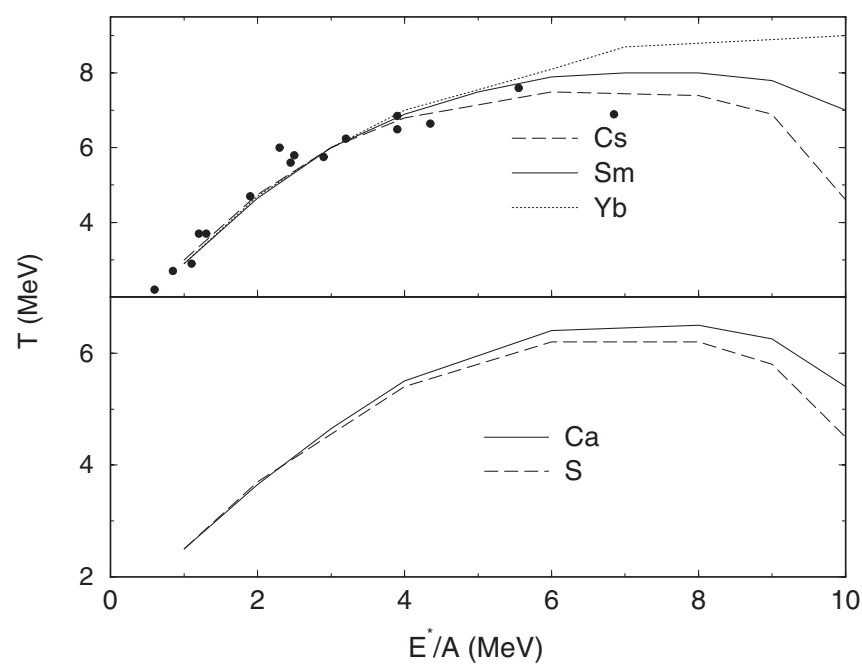

FIG. 6. Mononuclear caloric curves showing isospin effects for $A=150$ isobars (top panel) and $A=40$ isobars (bottom panel). Experimental data are as in Fig. 5. Information on the mass dependence of the caloric curves can be gained by comparing the curves of the pair of nuclei ${ }^{150} \mathrm{Sm}$ and ${ }^{40} \mathrm{~S}$ that have asymmetry $X \sim 0.2$, with those of the nuclei ${ }^{40} \mathrm{Ca}$ and ${ }^{150} \mathrm{Yb}$ that are symmetric or nearly symmetric. 


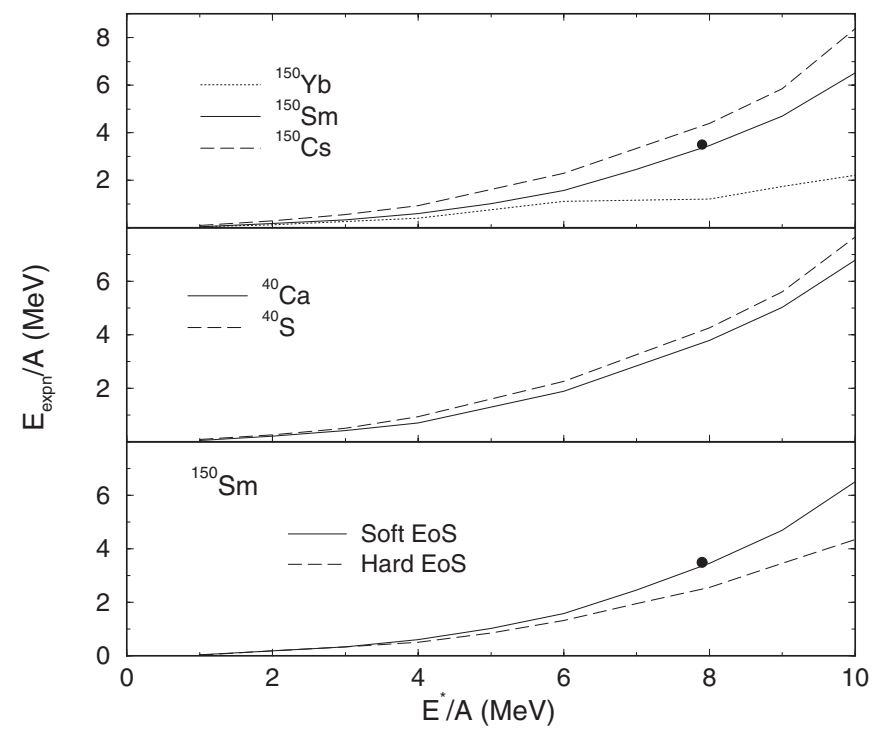

FIG. 7. Expansion energy as a function of total excitation energy for $A=150$ isobars (top panel) and $A=40$ isobars (middle panel). EOS dependence of the expansion energy for ${ }^{150} \mathrm{Sm}$ (bottom panel). Filled circles are experimental estimates [6].

the cost of thermal energy and hence there may be a density region in which the temperature may decrease with increasing excitation if the system expands much in pursuit of maximum entropy. In a simplistic model in Appendix $\mathrm{C}$, we show that this occurs in the density region $1 / 4<\rho / \rho_{0}<5 / 8$. It is also found that the more symmetric systems have lesser bends in the caloric curves (for $\mathrm{Yb}$, in the excitation energy range explored, the bend shows up only later at $E^{*} / A \sim 11 \mathrm{MeV}$ ), this is because they offer more resistance toward volume expansion, as already explained.

The expansion energy $E_{\text {expn }}$ constitutes a part of the total excitation. It is defined as $E_{\text {expn }}=E(\lambda, T)-E(\lambda=1, T)$. In Fig. 7, it is displayed as a function of $E^{*}$ for the different systems. In the bottom panel, the effect of the EOS on the expansion energy is shown for the nucleus ${ }^{150} \mathrm{Sm}$. It is seen that $E_{\text {expn }}$ decreases with stiffness of the EOS; the same is true for all other systems studied. The filled circle refers to an experimental estimate [6] for medium-mass nuclei $(A \sim 160-180)$. This is very close to our calculations with the softer EOS for the ${ }^{150} \mathrm{Sm}$ nucleus. The top and middle panels refer to calculated results for isobars with $A=150$ and 40 , respectively. As already stated, the more symmetric nuclei have effectively a harder EOS, this is reflected in the comparatively lower expansion energy for these nuclei. It is seen that in general, the expansion energy comprises a significant part of the total excitation.

The state with maximum entropy $S\left(\lambda_{\text {eq }}, T_{\text {eq }}\right)$ corresponds to the state with maximum probability. Other configurations with different values of the scale parameter $\lambda$ and temperature $T$ are also probable. Their probability is determined by the entropy profile $S(\lambda, T)$ at a fixed excitation energy:

$$
W(\lambda, T) \propto e^{S(\lambda, T)} .
$$

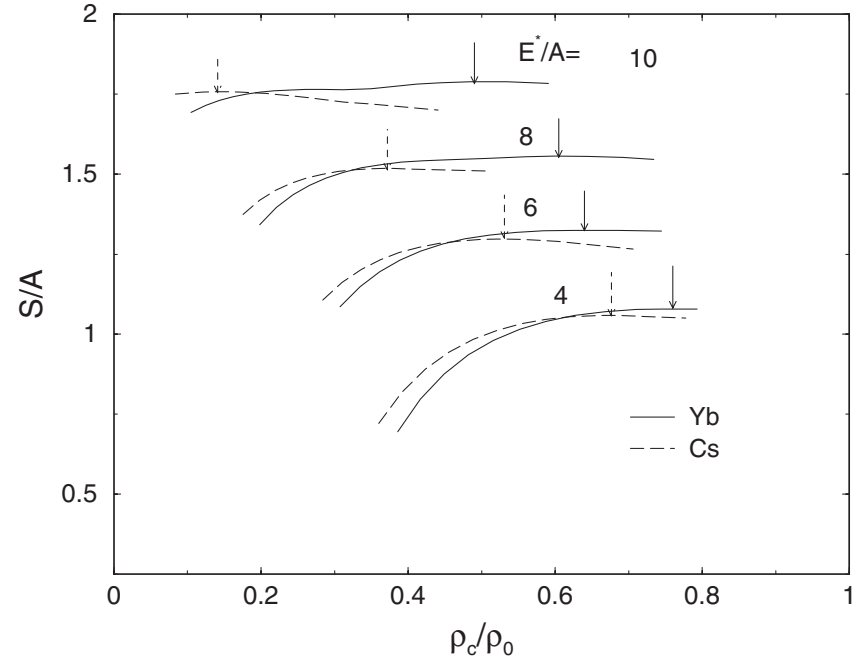

FIG. 8. Entropy profile at different fixed excitations as a function of the central density for the nuclei ${ }^{150} \mathrm{Yb}$ and ${ }^{150} \mathrm{Cs}$. Equilibrium configurations are marked by arrows, full ones for $\mathrm{Yb}$, and broken ones for Cs.

The $n$th moment of the central density $\rho_{c}$ is then

$$
\left\langle\rho_{c}^{n}(\lambda, T)\right\rangle=\frac{\int e^{S(\lambda, T)} \rho_{c}^{n}(\lambda, T) d \rho}{\int e^{S(\lambda, T)} d \rho} .
$$

Similarly, for the temperature $T$ one has

$$
\left\langle T^{n}(\lambda, T)\right\rangle=\frac{\int e^{S(\lambda, T)} T^{n}(\lambda, T) d T}{\int e^{S(\lambda, T)} d T} .
$$

Equations (49) and (50) allow one to calculate the mean and the variance of $\rho_{c}$ and $T$ at a fixed excitation. Figures 8 and 9 display the entropy profile for the systems ${ }^{150} \mathrm{Cs}$ and ${ }^{150} \mathrm{Yb}$ at different fixed excitations $E^{*} / A=4,6,8$, and $10 \mathrm{MeV}$ as a function of the central density (Fig. 8) and temperature (Fig. 9) of the expanded configurations. The arrows in the figures

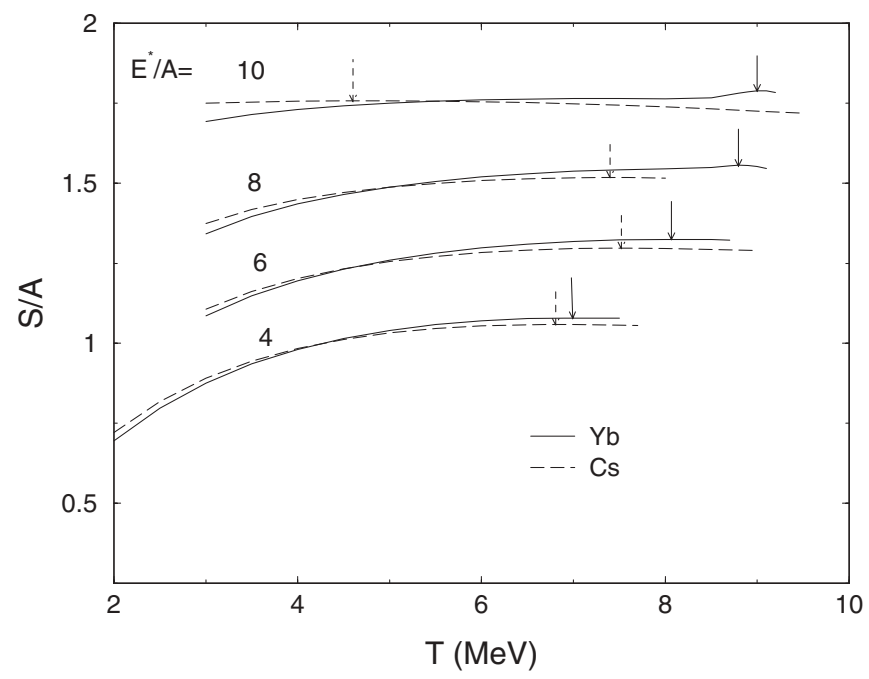

FIG. 9. Entropy profile at different fixed excitations as a function of temperature for the systems ${ }^{150} \mathrm{Yb}$ and ${ }^{150} \mathrm{Cs}$. Arrows have the same meaning as in Fig. 8. 


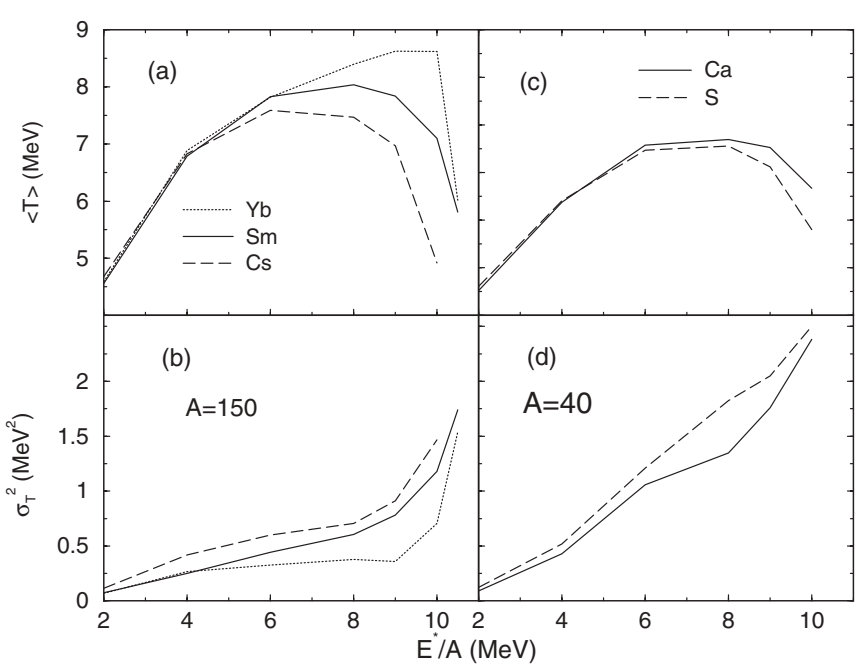

FIG. 10. Average temperature as a function of excitation energy in panels (a) and (c) for $A=150$ and $A=40$ isobars, respectively. Panels (b) and (d), corresponding variances in temperature.

indicate the configurations with the maximum probabilities, the full ones refer to $\mathrm{Yb}$ and the broken ones correspond to Cs. The entropy profiles are seen to flatten with excitation. This implies larger fluctuations at higher excitations.

For a thermodynamic system, the average and most probable (equilibrium) value of an observable are the same. For a finite system, however, they may differ. Experimentally, it is the average value that one measures. The average temperatures for the $A=150$ isobaric systems are displayed in panel (a) of Fig. 10; the corresponding quantities for ${ }^{40} \mathrm{Ca}$ and ${ }^{40} \mathrm{~S}$ are shown in panel (c). Panels (b) and (d) display the variances in temperature for the isobaric systems with $A=150$ and 40 , respectively. The equilibrium temperatures as shown in Fig. 6 are somewhat larger than the corresponding average values. Fluctuations build up with excitation. At a given excitation energy, the fluctuations for the lighter systems are comparatively higher, as expected. For the heavier systems, a sudden increase in fluctuation is observed beyond $E^{*} / A \sim$ $9 \mathrm{MeV}$, whereas the increase is relatively smooth for the lighter systems. In Fig. 11, the average values of the specific volume $v_{c}\left(=1 / \rho_{c}\right)$ in units of $v_{0}\left(=1 / \rho_{0}\right)$ and their variances for the systems considered are shown. The equilibrium volume is found to be somewhat less than the average value. As in the case of temperature, the fluctuations in volume rise smoothly up to an excitation energy $E^{*} / A \sim 9 \mathrm{MeV}$, beyond which the buildup is very sudden. This sudden buildup is more pronounced in comparison to that in temperature for both light and heavy systems. This large density fluctuation indicates that beyond $E^{*} / A \sim 9 \mathrm{MeV}$, the systems become unstable and break up into many pieces. It turns out that the negative branch of the heat capacity and the onset of large fluctuations occur at around the same excitation energy, indicating a possible close correlation between them.

Along with expansion, the excited nucleus may undergo deformation if that is profitable from entropy considerations. From the interplay of Coulomb and surface energies, a barrier is found along the deformation path; the barrier decreases with

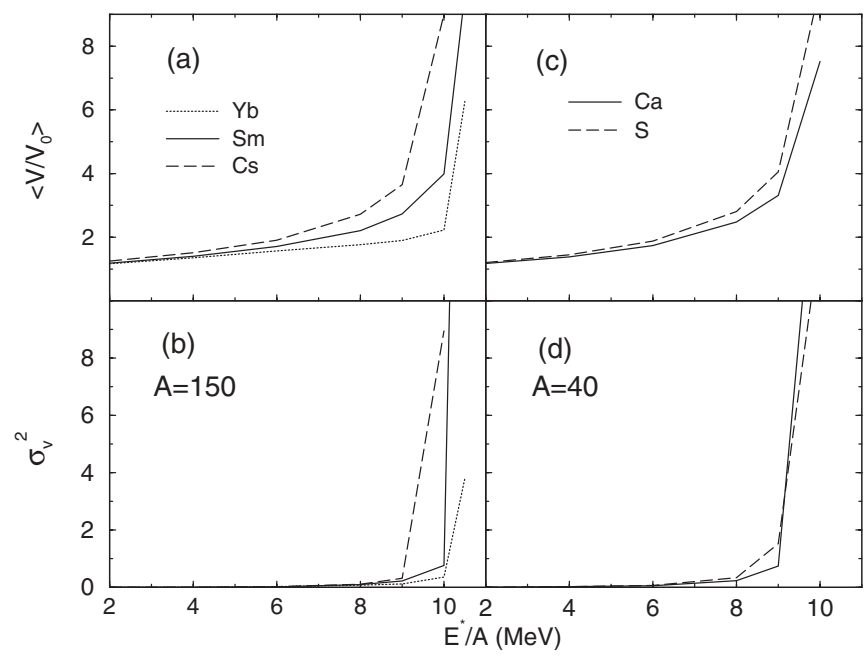

FIG. 11. Average values of the specific volumes and their variances for the different systems considered.

increasing temperature and decreasing density (expansion). At a particular temperature, for the deforming system, in our calculations the scale parameter $\lambda$ is taken such that the total excitation at the top of the barrier matches the given excitation $E^{*} / A$. This is repeated for different temperatures, and the maximum entropy among these different configurations is selected. If this entropy exceeds that for the expanded spherical equilibrium configuration, then deformation is favored leading to the fragmentation channel.

The extra entropy $\Delta S$ gained from deformation over that at a spherical equilibrium shape is displayed in the upper panels of Fig. 12 for the nuclei Sm and Cs as a function of excitation energy. As mentioned before, we considered a volume-conserving quadrupole deformation. For $E^{*} / A$ less than $\sim 4 \mathrm{MeV}, \Delta S$ is negative in the restricted deformation space chosen; it is thus seen that only above this excitation domain, fragmentation resulting from deformation is more

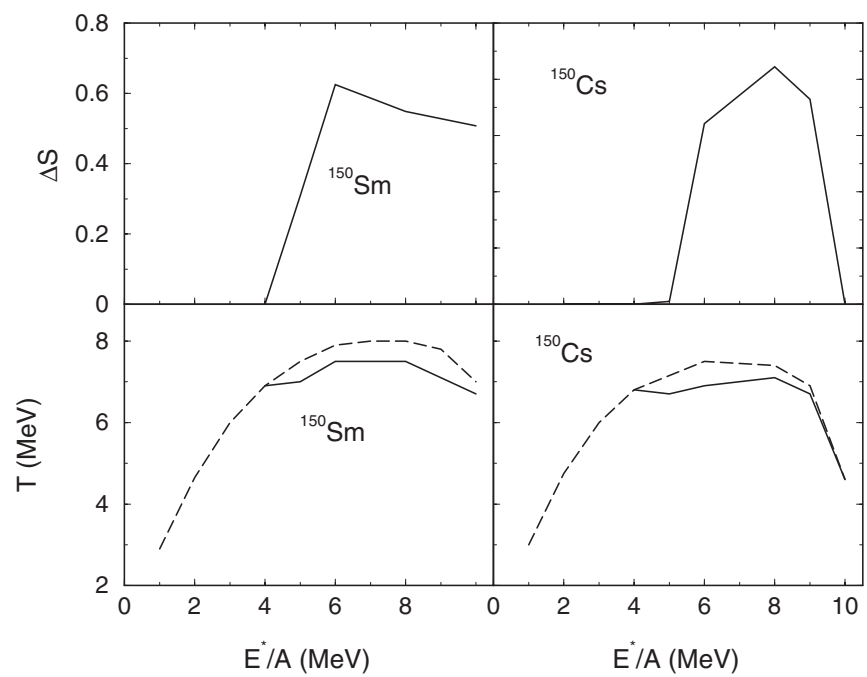

FIG. 12. Entropy gain $\Delta S$ from deformation as a function of $E^{*} / A$ for the systems ${ }^{150} \mathrm{Sm}$ and ${ }^{150} \mathrm{Cs}$ in upper panels. Caloric curves with (full line) and without (dashed line) deformation in lower panels. 
favorable. With deformation, the maximum entropy configuration occurs at a lower temperature at a given excitation; the corresponding caloric curves are shown in the bottom panels. In all the calculations, the oblate shapes are inhibited due to their lower entropy.

\section{CONCLUDING REMARKS}

In the present work, the gross features of an expanding mononuclear configuration at moderate and higher excitations have been dealt with in a semimicroscopic framework. A finite range, momentum- and density-dependent realistic effective interaction has been employed for our investigation. Renormalization of the nucleon mass coming from the coupling of the single-particle motion with the surface degrees of freedom is seen to play an important role in the correlation of entropy with excitation in a microcanonical formulation, this has been taken into account phenomenologically to avoid complexity. The density dependence in the interaction leaves room to vary the nuclear equation of state well within the ambits of accepted nuclear parameters. We have explored the influence of the EOS on mononuclear observables, and we find that at a fixed excitation, a nucleus with a softer EOS has more expansion, the corresponding expansion energy is found to be larger, and thus its effective temperature is comparatively lower.

The resultant mononuclear caloric curve and equilibrium density compare very well with the experimental data with the chosen softer EOS with nuclear incompressibility $K_{\infty}=$ $238 \mathrm{MeV}$, which is close to its well-accepted value. We have further studied the mass and isospin dependence on the mononuclear observables. For nuclei with the same asymmetry but higher mass, it is found that the system expands less and consequently has a higher temperature; this is also true for nuclei with the same mass but less asymmetry. All these observations could be explained from the fact that heavier and symmetric systems have effectively higher incompressibility.

In the experimentally constructed nuclear caloric curves $[39,40]$, a plateau is observed in the excitation energy range $\sim 3-8 \mathrm{MeV} / A$. This is indicative of infinite specific heat. In some analyses, a negative specific heat [41] in this energy domain is also suggested. These are pointers to a possible liquidgas phase coexistence. In our calculations, the plateau comes naturally from nuclear expansion, so also its bending (negative specific heat) at a relatively higher excitation. The fluctuations in temperature and volume build up with excitation. The rapid growth of these fluctuations at $E^{*} / A \sim 9 \mathrm{MeV}$ is suggestive of the instability of the mononuclear configuration against prompt multifragmentation. The occurrence of the negative heat capacity and the sudden growth of fluctuations at around the same excitation energy indicate a close correlation between them.

While expanding, the nuclear system may gain further entropy from fragmentation into a number of pieces, mostly from the generation of new surfaces. We have dealt with this in a simplistic way through a volume-conserving quadrupole deformation as a precursor to fragmentation. It is found that above $E^{*} / A \sim 4 \mathrm{MeV}$, the systems generally favor deformation. The evolution of the mononuclear configuration into a number of fragments may be treated through higher multipole deformation; it would be an involved task, but it is worth investigating.

\section{ACKNOWLEDGMENTS}

S.K.S. and J.N.D. acknowledge the financial support from CSIR and DST, Government of India, respectively. M.C. and X.V. acknowledge financial support from Grant No. FIS200503142 from MEC (Spain) and FEDER, and No. 2005SGR00343 from Generalitat de Catalunya.

\section{APPENDIX A: SINGLE-PARTICLE POTENTIALS}

The components of the single-particle potentials for the SBM interaction (1) entering in Eq. (3) can be written as [20]

$$
\begin{aligned}
& V_{\tau}^{0}\left(r_{1}\right)=-2 \pi a^{2} \int_{0}^{\infty}\left[1-d^{2}\left\{\rho\left(r_{1}\right)+\rho\left(r_{2}\right)\right\}^{n}\right] u\left(r_{1}, r_{2}\right) \\
& \times\left[C_{l} \rho_{\tau}\left(r_{2}\right)+C_{u} \rho_{-\tau}\left(r_{2}\right)\right] r_{2}^{2} d r_{2}+\frac{a^{2}}{\pi b^{2} \hbar^{3}} \\
& \times \int_{0}^{\infty} u\left(r_{1}, r_{2}\right)\left[C_{l}\left(2 m_{\tau, k}\left(r_{2}\right) T\right)^{5 / 2} J_{3 / 2}\left(\eta_{\tau}\left(r_{2}\right)\right)\right. \\
&\left.+C_{u}\left(2 m_{-\tau, k}\left(r_{2}\right) T\right)^{5 / 2} J_{3 / 2}\left(\eta_{-\tau}\left(r_{2}\right)\right)\right] r_{2}^{2} d r_{2}, \quad(\mathrm{~A} 1) \\
& V_{\tau}^{1}\left(r_{1}\right)=\frac{2 \pi a^{2}}{b^{2}} \int_{0}^{\infty} u\left(r_{1}, r_{2}\right)\left[C_{l} \rho_{\tau}\left(r_{2}\right)+C_{u} \rho_{-\tau}\left(r_{2}\right)\right] r_{2}^{2} d r_{2}, \\
& V_{\tau}^{2}\left(r_{1}\right)= \\
& 2 \pi n a^{2} d^{2} \sum_{\tau^{\prime}}\left\{\left[C_{l} \rho_{\tau^{\prime}}\left(r_{1}\right)+C_{u} \rho_{-\tau^{\prime}}\left(r_{1}\right)\right]\right. \\
&\left.\times \int_{0}^{\infty} \rho_{\tau^{\prime}}\left(r_{2}\right) u\left(r_{1}, r_{2}\right)\left[\rho\left(r_{1}\right)+\rho\left(r_{2}\right)\right]^{n-1} r_{2}^{2} d r_{2}\right\},
\end{aligned}
$$

with

$$
\rho(r)=\rho_{\tau}(r)+\rho_{-\tau}(r)
$$

and

$$
u\left(r_{1}, r_{2}\right)=\left[e^{-\left|r_{1}-r_{2}\right| / a}-e^{-\left(r_{1}+r_{2}\right) / a}\right] /\left(r_{1} r_{2}\right) .
$$

In these equations, if $\tau$ refers to proton, $-\tau$ refers to neutron, and vice versa. From the structure of Eq. (A3), it is seen that the rearrangement potential $V_{\tau}^{2}(r)$ is isospin independent.

The Coulomb single-particle potential is the sum of the direct and exchange contributions

$$
V_{c}\left(r_{1}\right)=V_{c}^{D}\left(r_{1}\right)+V_{c}^{\mathrm{Ex}}\left(r_{1}\right)
$$

where

$$
V_{c}^{D}\left(r_{1}\right)=\frac{2 \pi e^{2}}{r_{1}} \int_{0}^{\infty}\left[\left(r_{1}+r_{2}\right)-\left|r_{1}-r_{2}\right|\right] \rho_{p}\left(r_{2}\right) r_{2} d r_{2},
$$


and

$$
V_{c}^{\mathrm{Ex}}\left(r_{1}\right)=-e^{2}(3 / \pi)^{1 / 3} \rho_{p}^{1 / 3}\left(r_{1}\right) .
$$

\section{APPENDIX B: EQUIVALENCE OF SCALED AND CONSTRAINED DENSITY}

In this Appendix, we show the equivalence between the scaled density and the constrained density for a harmonic oscillator (HO) potential.

From Eqs. (7) and (8), the density at a finite temperature for a nucleus (ignoring the isospin) can be written in the form

$$
\rho(r)=\frac{8 \pi}{h^{3}}(2 m T)^{3 / 2} J_{1 / 2}(\eta),
$$

where $\eta=(\mu-V) / T, V$ being the single-particle potential. For small temperatures, $\eta$ is large. When $\mu<V, \eta$ is large negative and then $J_{1 / 2}(\eta) \sim e^{\eta}$, leading to vanishing density. When $\mu>V, \eta$ is large positive and then $J_{1 / 2}(\eta) \simeq \frac{2}{3} \eta^{3 / 2}$, and the density is given by

$$
\rho(r)=\frac{2}{3 \pi^{2}}\left(\frac{2 m}{\hbar^{2}}\right)^{3 / 2}(\mu-V)^{3 / 2} .
$$

For a harmonic oscillator potential with oscillator frequency $\omega, V=\frac{1}{2} m \omega^{2} r^{2}$ and the corresponding density is

$$
\rho(r)=B\left(\mu-\frac{1}{2} m \omega^{2} r^{2}\right)^{3 / 2},
$$

where $B=\frac{2}{3 \pi^{2}}\left(\frac{2 m}{\hbar^{2}}\right)^{3 / 2}$. The total number of particles for this density is

$$
A=\int \rho(r) d \mathbf{r}=\frac{2}{3}\left(\frac{\mu}{\hbar \omega}\right)^{3} .
$$

For the density given by Eq. (B3), following Eq. (19), the scaled density is

$$
\rho_{\lambda}(r)=B\left[\mu \lambda^{2}-\frac{1}{2} m\left(\omega \lambda^{2}\right)^{2} r^{2}\right]^{3 / 2} .
$$

It is seen that the chemical potential and the oscillator frequency are now scaled as

$$
\mu_{\lambda} \rightarrow \mu \lambda^{2} \quad \text { and } \quad \omega_{\lambda} \rightarrow \omega \lambda^{2} .
$$

The constrained Hamiltonian density constraining the particle number and the rms radius is given by

$$
\mathcal{H}(r)=\mathcal{E}^{\mathrm{kin}}(r)+\left(V-\alpha r^{2}\right) \rho_{\alpha}(r)-\mu_{\alpha} \rho_{\alpha}(r),
$$

where $\mathcal{E}^{\mathrm{kin}}$ is the kinetic energy density, $\mu_{\alpha}$ constrains the total number of particles, and $\alpha$ constrains the rms radius to given values $A$ and $R_{\alpha}$, respectively, so that

$$
A=\int \rho_{\alpha}(r) d \mathbf{r},
$$

and

$$
R_{\alpha}^{2}=\frac{1}{A} \int r^{2} \rho_{\alpha}(r) d \mathbf{r} .
$$

In the absence of the constraint on the rms radius, $\rho_{\alpha} \rightarrow \rho$ and $R_{\alpha} \rightarrow R$, the unconstrained radius.
In analogy to Eq. (B2), at low temperatures, the density corresponding to the Hamiltonian given by Eq. (B7) for a HO potential is written as

$$
\rho_{\alpha}(r)=B\left[\mu_{\alpha}-\frac{1}{2} m \omega^{2} r^{2}+\alpha r^{2}\right]^{3 / 2},
$$

which can be recast as

$$
\rho_{\alpha}(r)=B\left[\mu_{\alpha}-\frac{1}{2} m \omega_{\alpha}^{2} r^{2}\right]^{3 / 2},
$$

where $\omega_{\alpha}^{2}=\omega^{2}-2 \alpha / m$. Again, following Eq. (B4), the total particle number from Eq. (B8) is given by

$$
A=\frac{2}{3}\left(\frac{\mu_{\alpha}}{\hbar \omega_{\alpha}}\right)^{3} .
$$

From Eqs. (B9) and (B12), it then follows that

$$
\hbar \omega_{\alpha} R_{\alpha}^{2}=\frac{\hbar^{2}}{2 m} \frac{3}{2}\left(\frac{3 A}{2}\right)^{1 / 3} .
$$

This is also true for the unconstrained density $(\alpha=0)$ :

$$
\hbar \omega R^{2}=\frac{\hbar^{2}}{2 m} \frac{3}{2}\left(\frac{3 A}{2}\right)^{1 / 3} .
$$

From Eqs. (B13) and (B14),

$$
\hbar \omega_{\alpha}=\frac{R^{2}}{R_{\alpha}^{2}} \hbar \omega .
$$

Similarly, from Eqs. (B12) and (B15),

$$
\mu_{\alpha}=\mu \frac{R^{2}}{R_{\alpha}^{2}} .
$$

Therefore, the constrained density given by Eq. (B11) is rewritten as

$$
\rho_{\alpha}(r)=B\left[\mu \frac{R^{2}}{R_{\alpha}^{2}}-\frac{1}{2} m\left(\omega \frac{R^{2}}{R_{\alpha}^{2}}\right)^{2} r^{2}\right]^{3 / 2} .
$$

Identifying $\lambda$ as $R / R_{\alpha}$, one can see the equivalence of the scaled density given by Eq. (B5) with the constrained density.

\section{APPENDIX C: EOS DEPENDENCE OF EQUILIBRIUM DENSITY AND TEMPERATURE}

In this Appendix, we show in a simplistic model why the density and temperature at equilibrium are higher for a stiffer EOS at a given excitation $E^{*}$.

The expansion energy for the decrease of density from $\rho_{0}$ to $\rho$ is

$$
E_{\text {expn }}=\frac{K_{A}}{18}\left(1-\rho / \rho_{0}\right)^{2},
$$

which is the same form as suggested by Friedman [42]; $K_{A}$ is the incompressibility of the nucleus.

The thermal energy of a hot nucleus, in a Fermi-gas type model is

$$
E_{\text {ther }}=a T^{2},
$$


where the density dependence of the level density parameter is given as

$$
a=\frac{\pi^{2}}{4 \varepsilon_{F}(\rho)} .
$$

The latter can be written in the form

$$
a=b \rho^{-2 / 3},
$$

where $b$ is a constant. The total excitation energy is given by the sum of the expansion and the thermal energy,

$$
E^{*}=E_{\text {expn }}+E_{\text {ther }} .
$$

The entropy is given by $S(\rho, T)=2 a T=2 b \rho^{-2 / 3} T$. At equilibrium, $S$ is a maximum. Because of the constraint that the sum of the thermal and expansion energies is equal to the given $E^{*}$, the quantity to be maximized is

$$
\begin{aligned}
\tilde{S}(\rho, T)= & 2 b \rho^{-2 / 3} T \\
& -\frac{1}{v}\left[b \rho^{-2 / 3} T^{2}+\frac{K_{A}}{18}\left(1-\rho / \rho_{0}\right)^{2}-E^{*}\right],
\end{aligned}
$$

where $v$ is a Lagrange multiplier. Then $\partial \tilde{S} / \partial T=0$ yields $v=T$, and $\partial \tilde{S} / \partial \rho=0$ leads to

$$
b T^{2}=\frac{K_{A}}{6 \rho_{0}} \rho^{5 / 3}\left(1-\rho / \rho_{0}\right),
$$

resulting in

$$
E_{\text {ther }}=\frac{K_{A}}{6} \frac{\rho}{\rho_{0}}\left(1-\rho / \rho_{0}\right) .
$$

From Eqs. (C1) and (C8), the total excitation is

$$
E^{*}=\frac{K_{A}}{18}\left[1+\frac{\rho}{\rho_{0}}-2\left(\frac{\rho}{\rho_{0}}\right)^{2}\right] .
$$

Thus at a fixed $E^{*}$, if $K_{A}$ is higher, the quantity in the square bracket should be smaller. This is true if $\rho / \rho_{0}$ increases with $K_{A}$; in the simplistic model, this is satisfied only in the range $1 / 4<\rho / \rho_{0}<1$, which, however, covers almost the whole density range of our concern. From Eq. (C7),

$$
\frac{d T}{d E^{*}}=\frac{K_{A} \rho^{2 / 3}}{36 \rho_{0} b T}\left[\left(5-8 \frac{\rho}{\rho_{0}}\right) \frac{d \rho}{d E^{*}}\right] .
$$

Equation (C10) shows that $d T / d E^{*}$ is negative if the quantity in the square bracket is negative. From Eq. (C9), one finds that $d \rho / d E^{*}$ is negative in the density range $1 / 4<\rho / \rho_{0}<1$. In this schematic model, one thus finds that for $1 / 4<\rho / \rho_{0}<5 / 8$, the temperature decreases with excitation.
[1] C. Ishizuka, A. Ohnishi, and K. Sumiyoshi, Nucl. Phys. A723, 517 (2003).

[2] A. S. Botvina and I. N. Mishustin, Phys. Lett. B584, 233 (2004).

[3] B. A. Li and L. W. Chen, Phys. Rev. C 72, 064611 (2005).

[4] D. V. Shetty, S. J. Yennello, and G. A. Souliotis, in Proceedings of CAARI 2006, Fort Worth, TX, Aug. 20-25, 2006, arXiv:nuclex/0610019.

[5] S. Fritz et al., Phys. Lett. B461, 315 (1999).

[6] V. E. Viola, K. Kwiatkowski, J. B. Natowitz, and S. J. Yennello, Phys. Rev. Lett. 93, 132701 (2004).

[7] Ad. R. Raduta et al., Phys. Lett. B623, 43 (2005).

[8] J. B. Natowitz et al., Phys. Rev. C 66, 031601(R) (2002).

[9] W. Norenberg, G. Papp, and P. Rozmej, GSI preprint 2002-03, January, 2002B (unpublished).

[10] J. P. Bondorf et al., Phys. Rep. 257, 133 (1995).

[11] D. H. E. Gross, Rep. Prog. Phys. 53, 1122 (1990).

[12] S. Das Gupta and J. Pan, Phys. Rev. C 53, 1319 (1996).

[13] Ph. Chomaz and F. Gulminelli, Phys. Lett. B447, 221 (1999).

[14] L. G. Sobotka, R. J. Charity, J. Tõke, and W. U. Schröder, Phys. Rev. Lett. 93, 132702 (2004).

[15] J. N. De, S. K. Samaddar, X. Viñas, and M. Centelles, Phys. Lett. B638, 160 (2006).

[16] L. G. Sobotka and R. J. Charity, Phys. Rev. C 73, 014609 (2006).

[17] U. Lombardo and G. Russo, Phys. Rev. C 36, 841 (1987).

[18] K. T. R. Davies and S. E. Koonin, Phys. Rev. C 23, 2042 (1981).

[19] D. Bandyopadhyay, C. Samanta, S. K. Samaddar, and J. N. De, Nucl. Phys. A511, 1 (1990).

[20] J. N. De, N. Rudra, S. Pal, and S. K. Samaddar, Phys. Rev. C 53, 780 (1996).

[21] V. S. Uma Maheswari, D. N. Basu, J. N. De, and S. K. Samaddar, Nucl. Phys. A615, 516 (1997).

[22] N. Rudra and J. N. De, Nucl. Phys. A545, 608 (1992).
[23] B. Friedman and V. R. Pandharipande, Nucl. Phys. A361, 502 (1981).

[24] R. B. Wiringa, V. Fiks, and A. Fabrocini, Phys. Rev. C 38, 1010 (1988).

[25] P. Bonche, S. Levit, and D. Vautherin, Nucl. Phys. A436, 265 (1985).

[26] E. Suraud, Nucl. Phys. A462, 109 (1987).

[27] A. Bohr and B. R. Mottelson, Nuclear Structure, Vol. II (Benjamin, Reading, MA, 1975).

[28] R. Hasse and P. Schuck, Phys. Lett. B179, 313 (1986).

[29] P. F. Bortignon and C. H. Dasso, Phys. Lett. B189, 381 (1987).

[30] M. Prakash, J. Wambach, and Z. Y. Ma, Phys. Lett. B128, 141 (1983).

[31] S. Shlomo and J. B. Natowitz, Phys. Lett. B252, 187 (1990).

[32] J. N. De, S. Shlomo, and S. K. Samaddar, Phys. Rev. C 57, 1398 (1998).

[33] J. M. Eisenberg and W. Greiner, Microscopic Theory of the Nucleus (North Holland, Amsterdam, 1972), p. 394.

[34] W. D. Myers and W. J. Swiatecki, Ann. Phys. (NY) 55, 395 (1969).

[35] D. G. Ravenhall, C. J. Pethick, and J. M. Lattimer, Nucl. Phys. A407, 571 (1983).

[36] J. P. Blaizot, Phys. Rep. 64, 171 (1980).

[37] J. Treiner, H. Krivine, O. Bohigas, and J. Martorell, Nucl. Phys. A371, 253 (1981).

[38] M. M. Majumdar, S. K. Samaddar, N. Rudra, and J. N. De, Phys. Rev. C 49, 541 (1994).

[39] J. Cibor et al., Phys. Lett. B473, 29 (2000).

[40] J. Pochodzalla et al., Phys. Rev. Lett. 75, 1040 (1995).

[41] M. D’Agostino et al., Phys. Lett. B473, 219 (2000).

[42] W. A. Friedman, Phys. Rev. Lett. 60, 2125 (1988). 